\title{
A Novel Expert System for Building House Cost Estimation: Design, Implementation, and Evaluation
}

\author{
Yasser A. Nada \\ Chairman of Department of computer science, \\ Faculty of Computers and Information Technology, \\ Taif University - K S A,
}

\begin{abstract}
This paper introduces an expert system which demonstrates a new method for accurate estimation of building house cost. This system is simple and decreases the time, the effort, and the money of its beneficiaries. In addition, design and implementation of the proposed expert system are introduced. CLIPS 6.0 and C\# are used in implementation phase. Also, this expert system is programmed to be in a standalone package with a platform independency. Furthermore, the developed expert system is tested under several real cases. Finally, an initial evaluation of this expert system is carried out and a positive feedback is received from user's samples, which makes it robust and efficient.
\end{abstract}

Keywords-Expert System; Building House; CLIPS

\section{INTRODUCTION}

An expert system is a computer program designed to simulate the problem-solving behavior of a human who is an expert in a narrow domain or discipline. Expert Systems (ES), also called Knowledge Based System (KBS), are computer application programs that take the knowledge of one or more human experts in a field and computerize it so that it is readily available for use. Expert System makes easier for user to identify the describe symptoms like image bases or textual bases information as it is very difficult to describe in words. It can also be integrated with textual database which can be used for explanation purposes of basic terms and operations to confirm and to reach conclusion in some situations [1].

As a branch of artificial intelligence, an expert system has been widely used. An expert system shell greatly improves the efficiency of the construction of an expert system [2]. Become a computer systems mechanism profound impact on our daily lives as we see every day research and new projects for the use of computers to make life easier and save the experience, and ease the pressure borne by the people? The paper is a mix of the latest techniques presented in this section of the computer science related systems expert systems and decision support, the paper provides scientific material distinguished and easy for the user in the field of architecture in terms of the ability to set the vision and the perception of urban are easy and available, where the idea is based on the establishment of an expert system alternative to the architect be helpful to them in calculating the cost of the construction according to data entered from (The land area, the site of the Earth And,.. etc.), which provides immediate support to customers and make decisions based on information obtained by them and contained them within the scope of existing knowledge has it .
An expert system's knowledge base is traditionally encoded as a set of domain-specific rules. These rules are generally implications of the form:

IF a1 Ù a2 Ù ... Ù ak THEN ak+1 Ù ak+2 Ù ... Ù an, where the ai's are logical statements that are relevant to the system's problem domain. For example, in the context of soil science, the rule:

IF a soil is sandy and the level of humus is high THEN the soil is compact

The development of expert system is implemented in CLIPS programming environment (C Language Integrated Production System) $[3,4,5]$. This programming tool is designed to facilitate the development of software to model human knowledge or expertise. CLIPS program is used by reason of the flexibility, the expandability and the low cost.

The outline of the paper is as follows. Section2 problem recognition. Section3 presents the basic of building a house. Section 4 knowledge representation. Section 5 tree knowledge section 6 diagnosis process finally, working example and summarizes this paper.

\section{PROBLEM RECOGNITION}

We need to build expert system to presents the design and development of an expert system for Account the Cost of Building House $(\mathrm{ACBH})$. To distribute human expertise in this science.

\section{The BASIC OF BuILding A House}

- Choose a place of building a house

- Settlement of the land - soil quality

- Construction area

- Foundations and pillars.

- Types of foundations

- Finished Construction

- Types of buildings

- Types of fossils

- Internal planning for the home

- Determine the labor

-The numbers and types of housing required during the next twenty years in the Kingdom

\section{KNOWLEDGE REPRESENTATION}

The key problem is to find a KR (and a supporting reasoning system) that can make the inferences your application needs in time, that is, within the resource 
constraints appropriate to the problem at hand. This tension between the kinds of inferences and application "needs" and what counts as "in time" along with the cost to generate the representation itself makes knowledge representation engineering interesting.

There are representation techniques such as frames, rules, tagging, and semantic networks which have originated from theories of human information processing. Since knowledge is used to achieve intelligent behavior, the fundamental goal of knowledge representation is to represent knowledge in a manner as to facilitate inference (i.e. drawing conclusions) from knowledge [6,7].

Knowledge bases can be represented by production rules. These rules consist of a condition or premise followed by an action or conclusion (IF condition...THEN action).

\section{For example \\ If Land Area 400 \\ and work type foundation \\ and the number of floors 1 \\ and the number of room 4 \\ and the size of water tank small \\ Then cost 1,11000 SR}

TABLE 1

\begin{tabular}{|c|c|c|}
\hline Percent $\%$ & Number & Types of housing \\
\hline 4,23 & 13,812 & Villa \\
\hline 8,04 & 26,267 & Role at Villa \\
\hline 29,8 & 97,438 & Apartment \\
\hline 35,77 & 116,841 & Duplex \\
\hline 22,11 & 72,223 & Mtlasq \\
\hline & 326,581 & Total \\
\hline
\end{tabular}

A production rule system consists of

1- A set of rules.

2- Working memory that stores temporary data.

3- A forward or backward chaining inference engine.

\section{Simple Examples of Represent Rules for Expert System:}

FACT1 cost of the finishing normal1 equal 215,312SR
FACT2 cost of the finishing excellent equal 4,50342SR

FACT4 building foundations1 equal 252,684 SR

FACT5 building foundations 2 equal 4,45469 SR

FACT 6 Land area480

FACT 7 Land area400

FACT 8 the number of floor 2

FACT 9 the number of floor 1

FACT 10 water tank small

FACT 11 water tank medium

FACT 12 driver room NO

FACT 13 driver room YES

\section{RULE 1}

If the land area 480

and cost of building foundations 1

and the cost of finishing normal1

and the number of floor 2

Then the overall cost744,279 SR

RULE 2

If the land area400

and the number of floor 1

and water tank small

Then the cost of building foundations 6,50432 SR

RULE 3

If the Finishing Normal

and driver room $\mathrm{NO}$

and the number of floor 1

Then cost of the finishing 4,34762 SR

To prove the conclusion "the overall cost744, 279 SR" inference engine must prove all condition that leading to this conclusion. Condition can be found from asking user or from another Rule because this condition is conclusion in Rule.

\section{TREE KNOWLEDGE}

A decision tree (or tree diagram) is a decision support tool that uses a tree-like graph or model of decisions and their possible consequences, including chance event outcomes, resource costs. Decision trees are commonly used in operations research, specifically in decision analysis, to help identify a strategy most likely to reach a goal. Another use of decision trees is as a descriptive means for calculating conditional probabilities.

We give the example for land area 480 and $400 \mathrm{~m}$ square.

First: land area $400 \mathrm{~m} 2$ 


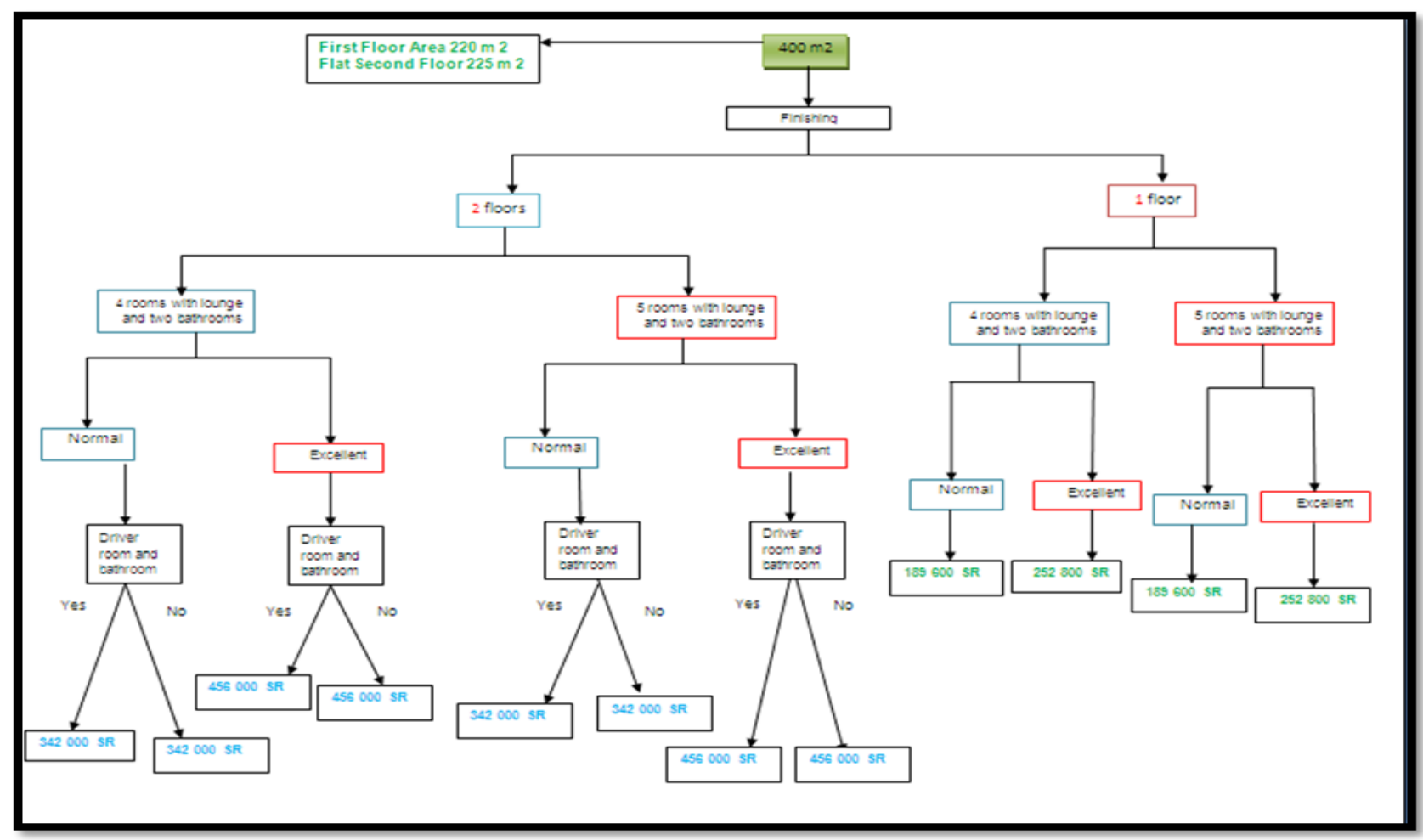

Fig. 1. Tree Knowledge - Foundation

\section{A. Foundational}

There are two options to the user in the Foundational, one or two floors.
If chosen for one floor, there are two cases before him to be the internal planning of the house 4 or 5 rooms with kitchen and two bathrooms.

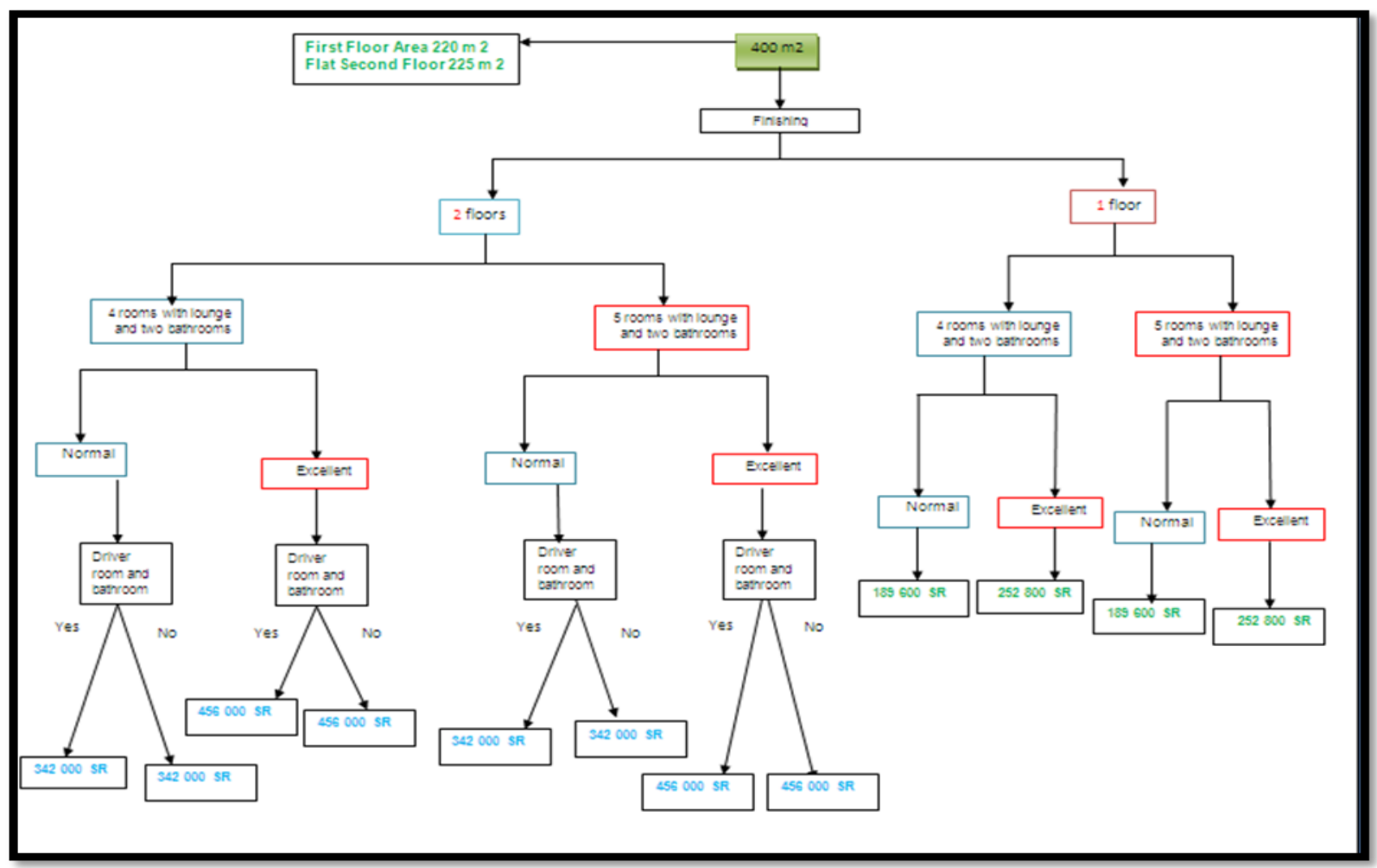

Fig. 2. Tree Knowledge - Finishing 


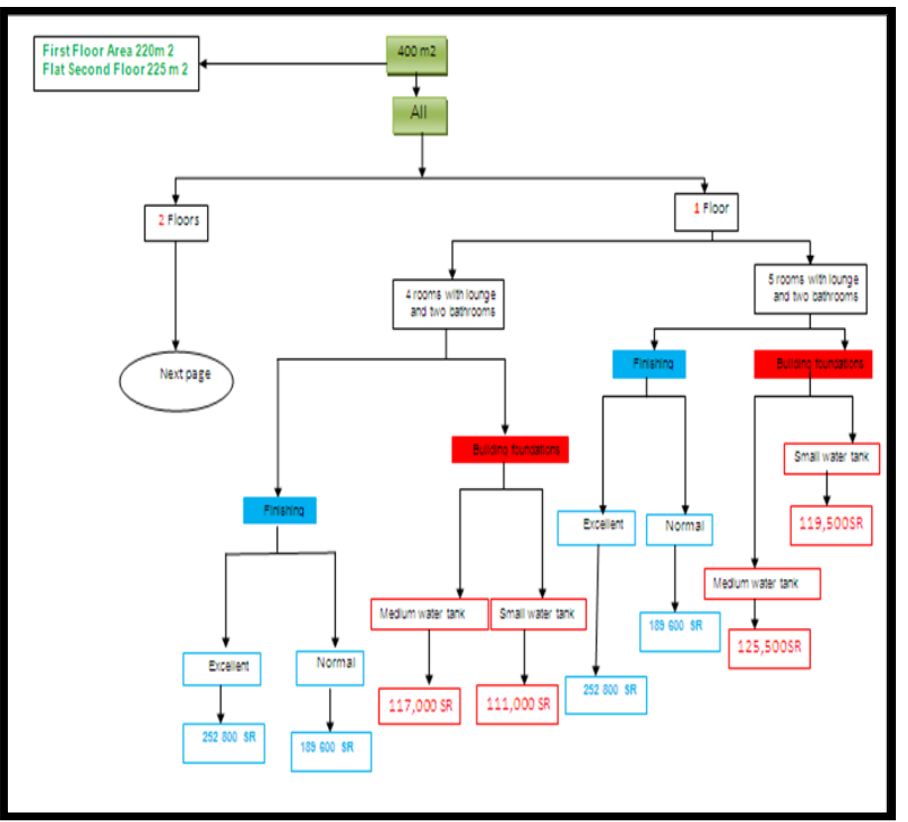

Fig. 3. Tree Knowledge - all- One
Then it will determine the size of tank water.

In the case of the user's choice 2 floors, will pass the same the previous options on one floor, but will start a driver with a bathroom extension, Show this explanation in figure (1).

\section{B. Finishing}

If the user chooses the option of calculating the cost of finishing, also given the choice between 1 or 2 floors. Then the internal planning 4 or 5 with kitchen and 2 bathrooms.

We have 2 type of finishing Normal, Excellent every Each type of them has a different cost from one another and are calculated cost them $\mathrm{m}^{2}$, Show this explanation in figure (2).

\section{C. $A L L$}

The user select choice All this mean (Foundational and finishing together), then calculate the cost at the same way previous,

Show this explanation in the following figures (3) and (4).

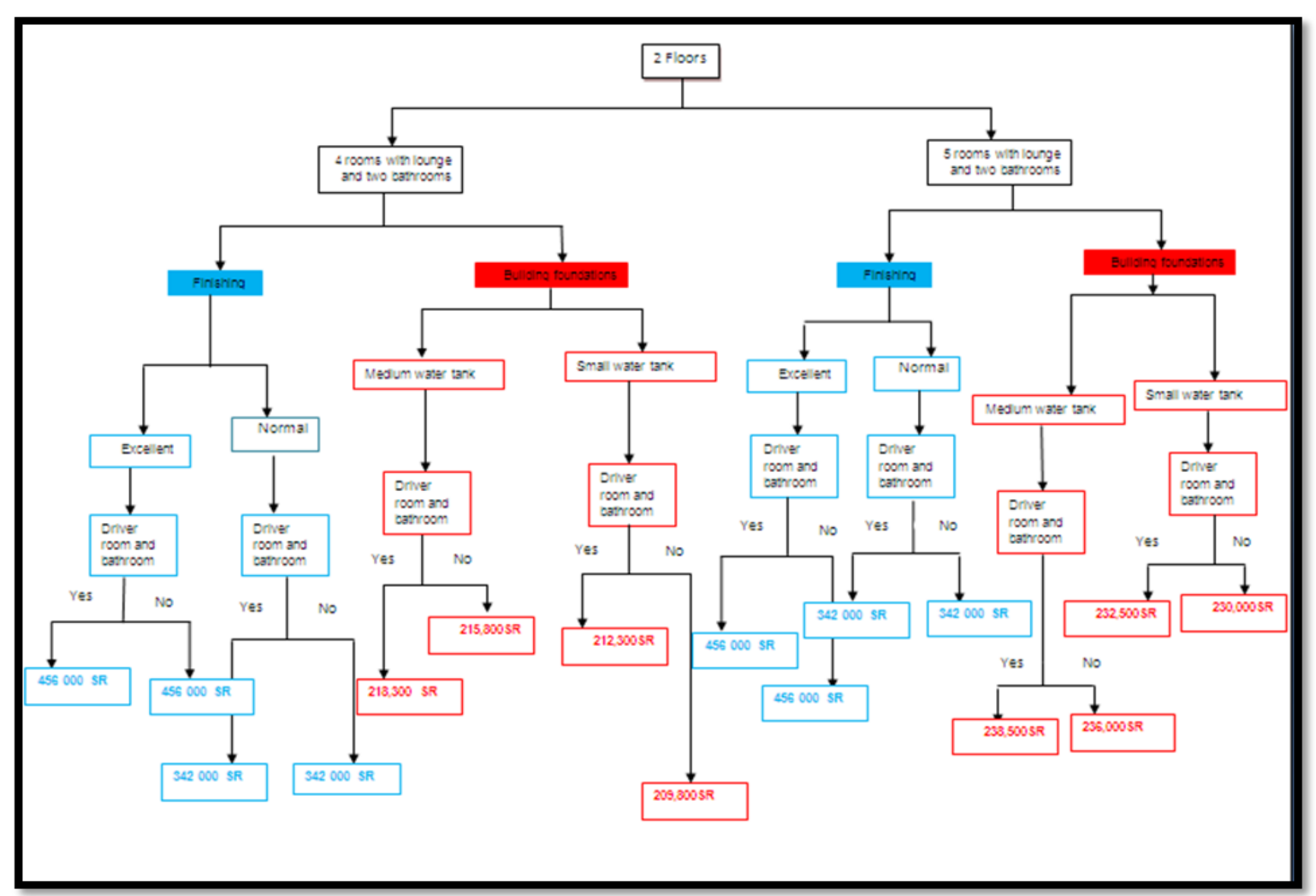

Fig. 4. Tree Knowledge - all- Two floors 


\section{Diagnosis PROCESS}

The expert system software adopted $\mathrm{C \#}$ to deal with the preparatory work, including the maintenance and management. All the status parameters, status values and solutions were obtained from the database access through $\mathrm{C \#}$, then past to CLIPS through interface functions that between C\# and CLIPS, lastly diagnosed by the program that was built by CLIPS, and meanwhile, the inference information and result were past to and displayed on the interface module, which was programmed by $\mathrm{C \#}$.

\section{Expert System Shell}

CLIPS keep in memory a fact list, a rule list, and an agenda with activations of rules. Facts in CLIPS are simple expressions consisting of fields in parentheses. Groups of facts in CLIPS, usually follow a fact-template, so that to be easy to organize them and thus design simple rules that apply to them. Our expert system contains 100 CLIPS rules. Below, we present the rules for $(\mathrm{ACBH})$.

\section{Working Example}

We can represent this rule using our representation as follow:

Some instruction in the Clips

defrule work_type_process

$=>$

(printout $\mathrm{t}$ "foundation, finish, all" crlf)

(bind ?answer (read idata))

(assert(work_type ?answer))

(printout t ?work_type)

$\Rightarrow$

(defrule area_process

(printout t "land area 400 or 480 ?" crlf)

(bind ?answer (read idata))

(assert(area ?answer)))

(defrule f1

(work_type foundation)(area 480)(floor one)(rooms five )(small tank)

$=>$

(printout odata "122342" crlf))

(defrule $\mathrm{f} 2$

(work_type foundation)(area 480) (floor one)(rooms five)(medium tank)

$$
=>(\text { printout odata "128342" crlf)) }
$$

Figures 5,6,7,8,9 and 10 present some samples from the proposed expert system forms and menus.

\section{After Execution}

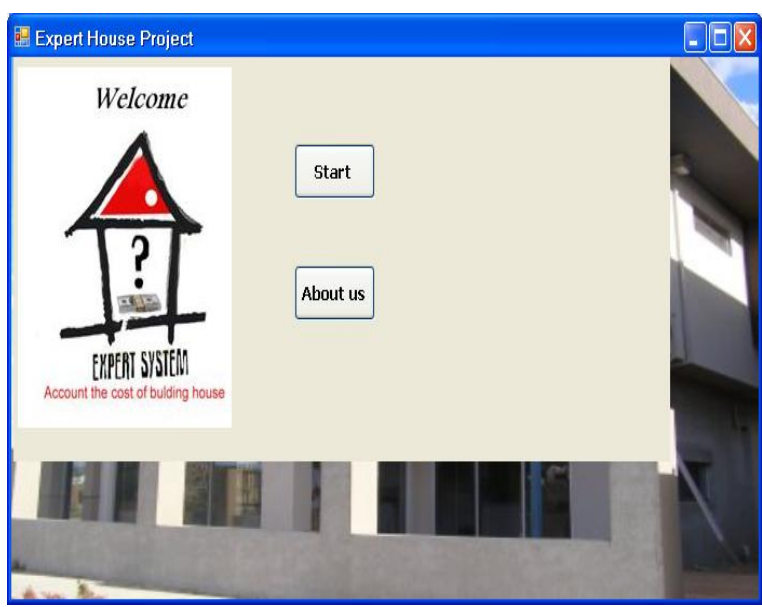

Fig.5. Start-up program

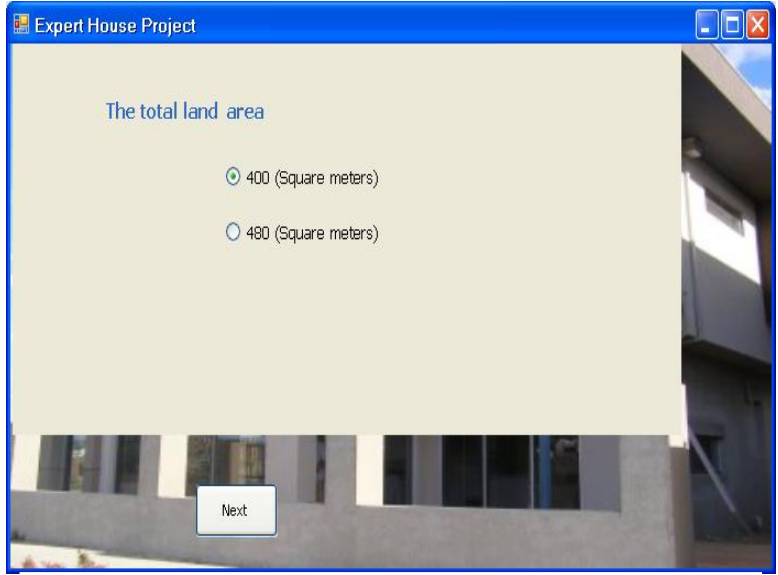

Fig.6. Second screen chose the land area

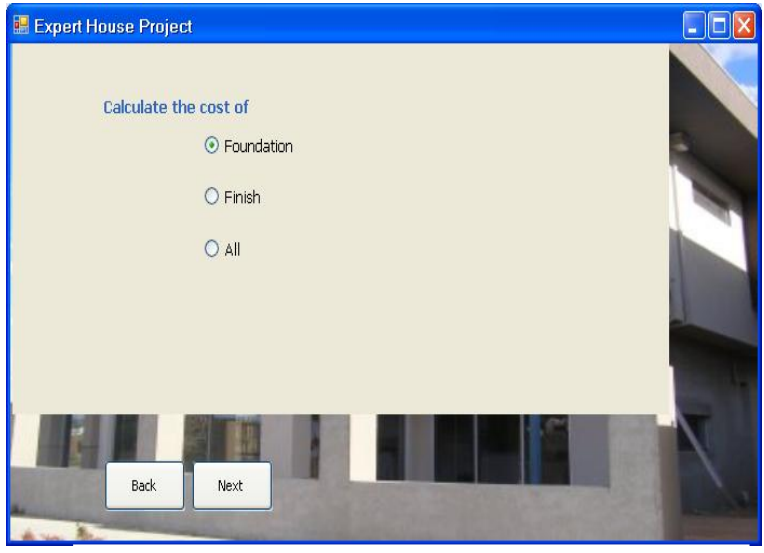

Fig.7. choose the type of work 


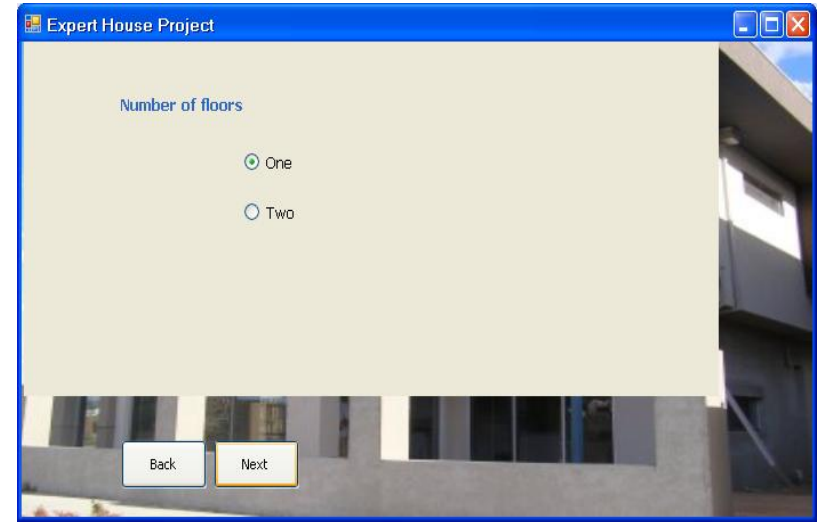

Fig.8. choose the number of floors

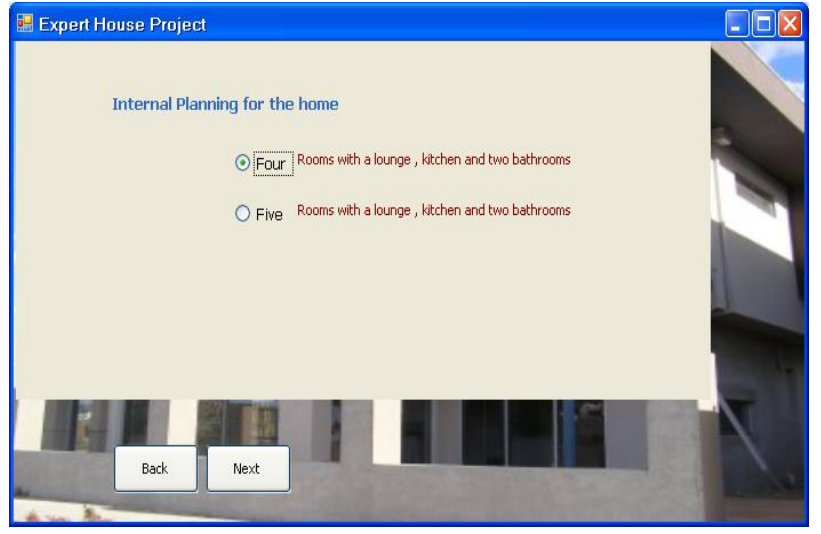

Fig.9. choose the number of rooms

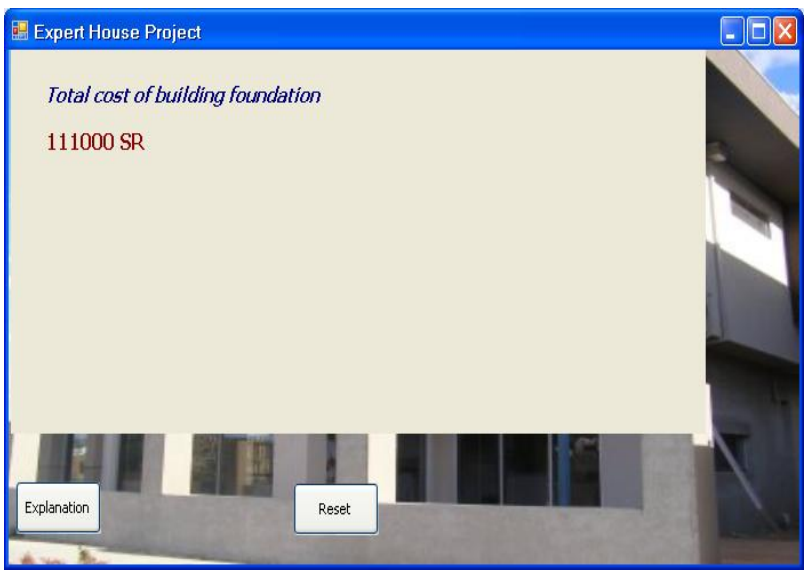

Fig.10. the result of cost SR

\section{CONCLUSIONS}

In this paper, the design of an expert system for estimating the Cost of building house is introduced. The expert system is implemented using Clips to build a knowledge base and C \# to design a foreground interface. The developed expert system interface is used to receive information from users and handle it under several cases. Accordingly, it returns an accurate estimation to the user. The proposed expert system is included in one executable standalone package. In addition, the proposed expert system test proves that it simple, accurate, powerful, and flexible.

\section{REFERENCES}

[1] Khumukcham R., Shikhar Kr. S, "JESS Based Expert System Architecture For Diagnosis Of Rice Plant Diseases: Design And Prototype Development", 2013 4th International Conference on Intelligent Systems, Modeling and Simulation, P 674-676, 2013.

[2] Qi Ming Cui, Sheng Wei Liu, Rong Xing Liu, and Zhi Rai Wang,"Application Research of the Production System type Expert System Shell Pro/3 under Smart Grid", IEEE PES ISGT ASIA 2012.

[3] Giarranto J. C., "CLIPS User's Guide", Version 6.22, 1998.

[4] Jackson, P., "Introduction to Expert Systems", Harlow, England: Addison Wesley Longman. Third Edition, 1999.

[5] Da-peng Tan, Shi-ming Ji Shu-ting Chen, "Continuous Casting Slag Detection Expert System Based on CLIPS", 2010 Ninth International Symposium on Distributed Computing and Applications to Business, Engineering and Science, IEEE, 2010.

[6] George F. Luger and William, A. Stubblefield; " Artificial Intelligence and the Design of Expert Systems", The Benjamin/ Cummings publishing Co., Inc., 1989.

[7] Keith D., "The Essence of Expert Systems", Prentice Hall, 2000.

\section{AUTHORS BIOGRAPHY}

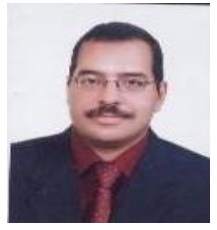

DR. Yasser Ahmed Nada Was born in Ismailia, Egypt, in 1968. He received the BSc degree in pure Mathematics and Computer Sciences in 1989 and MSc degree for his work in computer science in 2003, all from the Faculty of Science, Suez Canal University, Egypt. In 2007, he received his Ph.D. in Computer Science from the Faculty of Science, Suez Canal University, Egypt. From September 2007 until now, he worked as Assistant Professor of computer science. Chair, Department of computer science, Faculty of Computers and Information Technology, Taif University, KSA. His research interests include Expert Systems, Artificial Intelligence, Object Oriented Programming, Computer Vision, and Genetic. 